\title{
PROBABILIDADE DE CAIR E MEDO DE QUEDAS APÓS OFICINA DE EQUILÍBRIO EM IDOSOS PRATICANTES DE ATIVIDADE FÍSICA
}

\author{
PROBABILITY OF FALLING AND FEAR OF FALLING AFTER BALANCE \\ WORKSHOP IN ELDERLY PRACTIONERS OF PHYSICAL ACTIVITY
}

\section{Fabiana Ribeiro dos Anjos ${ }^{{ }^{*}}$, Andréa Kruger Gonçalves ${ }^{b^{*}}$, Eliane Mattana Griebler ${ }^{b^{*}}$, Eduardo Hauser ${ }^{r^{*}}$, Rayane Brum de Fraga ${ }^{\mathrm{d}^{*}}$, Lilian Benin ${ }^{\mathrm{e}^{*}}$, Ângelo José Gonçalves Bós ${ }^{\mathrm{f}^{* *}}$, Adriane Ribeiro Teixeira ${ }^{{ }^{*}}$}

\author{
afabrianjos@yahoo.com.br, bandreakg@terra.com.br, cehauser07@gmail.com, dray_fraga@hotmail.com, elililorenzatto@hotmail.com, \\ fangelo.bos@pucrs.br, gadriane.teixeira@gmail.com \\ *Universidade Federal do Rio Grande do Sul - Porto Alegre (RS), Brasil \\ **Pontifícia Universidade Católica do Rio Grande do Sul - Porto Alegre (RS), Brasil
}

Data de entrada do artigo: 03/05/2014

Data de aceite do artigo: 23/09/2014

\section{RESUMO}

Introdução: Quedas são eventos frequentes em idosos, causando restrição de participação social, principalmente pelo medo de novas quedas. Objetivo: Verificar o risco de quedas e a preocupação de cair em idosos praticantes de atividade física antes e após oficina de equilíbrio. Materiais e Métodos: Os idosos foram avaliados antes e após a realização da oficina, que foi desenvolvida durante seis meses, duas vezes por semana. Para a avaliação da probabilidade de cair foi utilizado o teste do alcance funcional anterior. A preocupação em cair foi avaliada por meio do Falls Efficacy Scale - International (FES-I). Resultados: Avaliou-se 17 idosos, de ambos os gêneros, com média de idade de 75,5 $\pm 8,5$ anos. Os valores médios pré e pós-oficina foram, respectivamente, de $34,2 \pm 6,5 \mathrm{~cm}$ e $36 \pm 4 \mathrm{~cm}$ no alcance funcional $(\mathrm{p}=0,09)$ e de $22,8 \pm 4,8$ pontos e $23,5 \pm 4,7$ no FES-I $(p=0,19)$. Conclusáo: Verificou-se que a probabilidade de quedas e a preocupação em cair não foram modificadas significativamente com a oficina, provavelmente porque os idosos eram praticantes de atividade física orientada.

Palavras-chave: Idoso; acidentes por quedas; equilíbrio postural.

\section{ABSTRACT}

Introduction: Falls are frequent events in the elderly, causing restriction of social participation, especially by fear of further falls. Objective: To assess the risk of falls and the fear of falling in elderly practitioners of physical activity before and after balance workshop. Materials and Methods: Subjects were evaluated before and after the workshop, which was developed for six months, twice a week. The Functional Reach Test (FES-I) was used for the evaluation of the probability of falling. The fear of falling was assessed using the Falls Eficacy Scales - International (FES-I). Results: We evaluated 17 elderly of both genders, with a mean age of $75.5 \pm 8.5$ years. The mean values pre and post-workshop were, respectively, 34.2 \pm 6.5 and $36 \pm 4 \mathrm{~cm}$, in the Functional Reach Test $(\mathrm{p}=0.09)$ and $22.8 \pm 4.8$ points and $23.5 \pm 4.7$ points in FES-I $(\mathrm{p}=0.19)$. Conclusion: It was found that the likelihood of falls and fear of falling were not significantly modified with the workshop, probably because the elderly were practitioners of guided physical activity.

Keywords: Elderly; accidental falls; postural balance. 


\section{Introdução}

O mundo está passando por um período de transição demográfica. O envelhecimento populacional ocorre em todos os países, mas seus efeitos são mais rápidos e abruptos nos países em desenvolvimento ${ }^{1,2}$. Dados do censo de 2010 evidenciam que a população idosa no Brasil era 20,6 milhóes, contra 14,5 milhôes em $2000^{3}$. $\mathrm{O}$ rápido crescimento populacional deste grupo etário exige conhecimentos e investimentos para a manutenção de sua qualidade de vida. As quedas estão entre os fatores que podem comprometer seriamente a qualidade de vida, a independência e a capacidade funcional dos idosos. Elas são provocadas por eventos multifatoriais, sendo os distúrbios de equilíbrio os principais fatores ${ }^{4}$.

$\mathrm{O}$ envelhecimento gera modificaçóes estruturais e funcionais, comprometendo todos os componentes do controle postural, sensorial, efetor e o processamento central. O equilíbrio é composto pela integração das informaçóes dos sistemas visual, sensorial e proprioceptivo, além da função vestibular e neuromuscular, permitindo a manutenção do controle postural ${ }^{5}$. $\mathrm{O}$ avanço da idade é diretamente proporcional à presença de múltiplos sintomas otoneurológicos, tais como vertigem e outras tonturas, perda auditiva, zumbido, alteraçôes do equilíbrio corporal, distúrbios da marcha, quedas ocasionais, entre outros ${ }^{6,7}$.

A queda é um evento acidental, frequente e limitan$t^{8,9}$. Tal evento gera insegurança e é fonte significativa de desabilidade e morte em pessoas idosas ${ }^{10}$. O medo de cair pode levar idosos a evitarem certas atividades ${ }^{11}$, causando limitaçôes, mas também pode ser uma prevenção, já que o idoso toma mais cuidado para não se expor a riscos ${ }^{12}$.

A morbidez, morte, hospitalização, institucionalização e a deterioração funcional podem ser ocasionadas pelas quedas. Estas também têm consequências que influenciam de maneira direta no dia a dia do idoso, como a restrição das atividades devido às dores decorrentes de fraturas, o medo de cair, o declínio da saúde, incapacidade e até mesmo atitudes protetoras de familiares ${ }^{8,10,14}$.

Idosos com comprometimento funcional do equilíbrio que buscam uma reabilitação precoce têm maior facilidade de prevenir a evolução e de recuperar a perda funcional incipiente, considerando que, com o tempo, seu corpo começa a responder de forma mais lenta e não há força muscular para controlar os movimentos. Com isso, amenizam e evitam que as limitações funcionais possam progredir e culminem em queda, atingindo consequências que podem variar entre a incapacidade $\mathrm{e}$ a morte $e^{15,16}$.

Estudos realizados no país evidenciam que a realização de exercícios psicomotores e prática de atividade física orientada podem auxiliar os idosos na melhora do equilíbrio e, consequentemente, na prevenção de quedas ${ }^{17,18}$, além de diminuir a preocupação em cair ${ }^{19}$. Desperta interesse, contudo, o impacto de uma oficina de prevenção de quedas com a realização de exercícios físicos específicos em idosos que praticam atividade física orientada regularmente.

Assim, o objetivo deste estudo foi verificar a probabilidade de quedas e o medo de cair em idosos praticantes de exercício físico pré e pós-participação em oficina de equilíbrio.

\section{Metodologia}

O estudo teve delineamento intervencionista, quantitativo e descritivo. A amostra foi escolhida por conveniência, sendo composta por idosos praticantes de exercício físico e participantes de uma oficina de equilíbrio.

Foram incluídos indivíduos com idade igual ou superior a 60 anos, praticantes de exercício físico em projeto de extensão na universidade. Foram excluídos os indivíduos que não aceitaram participar da pesquisa voluntariamente, não assinaram o Termo de Consentimento Livre e Esclarecido ou os que apresentaram histórico de doenças neurológicas, motoras, cognitivas ou psiquiátricas. Houve exclusão, também, dos idosos que apresentaram frequência inferior a $75 \%$ nas atividades desenvolvidas nas oficinas.

A coleta de dados foi feita em duas fases. Na primeira fase foi utilizado um questionário sócio-demográfico, contendo informaçóes sobre os participantes (idade, sexo, doenças, medicamentos, entre outros). A seguir, era aplicado o instrumento Falls Efficacy Scale International (FES-I) na versão adaptada e validada para o português brasileiro para avaliar o medo de quedas ${ }^{20}$. Este instrumento é composto por 16 questôes relacionadas ao medo de cair durante a realização de atividades diárias. Para cada situação o idoso deveria assinalar a preocupaçáo em cair, sendo apresentadas as seguintes opçóes: nem um pouco preocupado em cair (1 ponto); um pouco preocupado em cair (2 pontos); muito preocupado em cair (3 pontos) e extremamente preocupado em cair (4 pontos). A pontuação mínima é de 16 pontos e a máxima de 64 pontos. Quanto maior a pontuação, maior o medo de cair ${ }^{20}$.

Para o teste de alcance funcional anterior, uma fita métrica foi fixada à parede, paralela ao chão, na altura do acrômio de cada indivíduo. Os indivíduos foram orientados a posicionarem-se paralelamente à parede sem encostar-se à ela, com os pés paralelos e descalços, numa posição confortável. O membro superior foi posicionado com os ombros flexionados a $90^{\circ}$, cotovelos estendidos, punhos em posição neutra e mão espalmada. A seguir, eram orientados a inclinar-se para frente $o$ 
máximo possível e manter a posição por três segundos sem retirar os calcanhares do chão, perder o equilíbrio ou dar um passo. Caso isto ocorresse, a tentativa era desconsiderada, e uma nova tentativa era realizada. Tendo como parâmetro o terceiro metacarpo do indivíduo, foi registrado, em centímetros, o deslocamento anterior sobre a fita métrica. Deslocamentos menores do que 15,2 $\mathrm{cm}$ indicam fragilidade do paciente e risco de quedas quatro vezes maior em relação a quem atingiu mais de $25,4 \mathrm{~cm}$ (alta probabilidade de queda). Quem atingiu medidas entre 15,2 e $25,4 \mathrm{~cm}$ correspondeu a duas vezes mais chance de ter risco de queda (média probabilidade de queda). Valores superiores a $25,4 \mathrm{~cm}$ indicam baixa probabilidade de queda ${ }^{21}$.

Ao final desta primeira etapa de avaliação, foi iniciada a oficina de equilíbrio, que teve a duração de seis meses, com atividades de 50 minutos por duas vezes na semana. A oficina incluiu atividades visando exercícios de força com ênfase na musculatura dos membros inferiores, exercícios de alongamento e relaxamento, além do aquecimento e exercícios para controle do ajustamento (preensão plantar; variação de apoios, alturas e tipos de pisos; treino proprioceptivo; deslocamento em diferentes direçóes). A evolução das oficinas era realizada a partir do aumento do nível de exigência nos exercícios, seja manipulando o grau de dificuldade de coordenaçáa, como o aumento de carga nos exercícios de força, ou ainda o grau de amplitude articular nos exercícios de alongamento.

A estrutura das aulas foi composta por aquecimento articular inicial, seguido de exercícios para a estimulação das capacidades citadas por meio de circuito ou atividades específicas na parte principal. Na parte final da aula realizava-se o alongamento seguido de relaxamento.

Após o período de seis meses, foram realizadas as reavaliaçóes, incluindo os mesmos instrumentos utilizados na primeira etapa do estudo.

Os dados obtidos foram inseridos na planilha do software Excel e analisados estatisticamente através do programa Epi Info, versão 6.0. Foi calculada a média e desvio padrão da pontuação obtida no questionário e os valores absolutos e relativos da probabilidade de queda. Para a comparação entre os resultados (pré e pós-oficina), foi utilizado o teste t-Student para amostras pareadas, sendo considerados significativos valores de $\mathrm{p} \leq 0,05$.

O projeto desta pesquisa foi aprovado pelo Comitê de Ética em Pesquisa do Instituto de Psicologia da UFRGS, sob protocolo número 21629.

\section{Resultados}

A amostra inicial foi composta por 29 idosos. Devido às faltas, originadas pelos mais diversos motivos (doenças, viagens, mudanças climáticas), foram excluídos 12 idosos, sendo que 17 finalizaram as oficinas. A idade dos participantes variou entre 62 e 89 anos (média de $75,5 \pm 8,57$ anos), sendo 16 do sexo feminino $(94,1 \%)$. Praticavam ginástica, hidroginástica, jogging aquático e atividades físicas na oficina Promovendo a Autonomia e Independência Funcional (PAIF). Cada idoso praticava um tipo de atividade física e oficina de equilíbrio em horários distintos.

Na Tabela 1 são demonstrados os valores obtidos nos instrumentos pré e pós-oficina de equilíbrio. Verificouse que não houve diferença significativa entre as duas etapas.

Tabela 1: Pontuação obtida pelos indivíduos no Teste do Alcance Funcional e no Instrumento FES-I

\begin{tabular}{lcccc}
\hline & \multicolumn{2}{c}{$\begin{array}{c}\text { Alcance funcional } \\
(\mathbf{c m})\end{array}$} & \multicolumn{2}{c}{ FES-I } \\
\hline & Pré & Pós & Pré & Pós \\
Mínimo - máximo & $23-47$ & $24-46$ & $16-34$ & $18-34$ \\
Média (DP) & 34,2 & 36,0 & 22,8 & 23,5 \\
& $(8,57)$ & $(7,41)$ & $(4,82)$ & $(4,71)$ \\
Valor de $\mathrm{p}^{*}$ & \multicolumn{2}{c}{0,09} & \multicolumn{2}{c}{0,19} \\
\hline
\end{tabular}

cm - centímetros; ${ }^{*}$ Teste-T para amostras pareadas

$\mathrm{Na}$ Tabela 2 são colocados os resultados do teste do alcance funcional considerando a probabilidade de quedas. Verificou-se que a maior parte dos idosos apresentava baixa probabilidade de queda, tanto no período pré quanto no período pós-oficina de equilíbrio.

Tabela 2: Distribuição dos idosos, de acordo com a probabilidade de queda pré e pós-participação na oficina de equilíbrio

\begin{tabular}{lcccc} 
& \multicolumn{2}{c}{ Pré-oficina } & \multicolumn{2}{c}{ Pós-oficina } \\
& n & $\%$ & n & $\%$ \\
\hline $\begin{array}{l}\text { Média } \\
\text { probabilidade }\end{array}$ & 1 & 5,89 & 1 & 5,89 \\
$\begin{array}{l}\text { de queda } \\
\text { Baixa } \\
\text { probabilidade } \\
\text { de queda }\end{array}$ & 16 & 94,1 & 16 & 94,1 \\
\hline
\end{tabular}

\section{Discussão}

Este estudo foi realizado com o objetivo de verificar a probabilidade de quedas e a preocupação de cair em idosos praticantes de exercício físico pré e pós-participação em oficina de equilíbrio.

Os dados referentes à análise do alcance funcional anterior evidenciam que os valores obtidos, tanto no período pré quanto no período pós-oficina, foram elevados, sendo que somente um idoso apresentou média 
probabilidade de queda. Os demais apresentaram baixa probabilidade de queda.

Considerando os valores obtidos no teste do alcance funcional, verificou-se que foram superiores aos obtidos por outros pesquisadores, que também avaliaram idosos praticantes de atividades físicas ${ }^{22,23,24}$.

Os resultados obtidos nas duas fases foram semelhantes aos de outro estudo nacional. Essa outra pesquisa, contudo, destaca que houve um aumento significativo quando comparados os dados pré e pós-treinamento. A diferença pode ser explicada pelo fato de que os idosos avaliados no estudo citado participaram de atividades físicas por um período maior do que o proposto em nossa pesquisa ${ }^{21}$. Além disso, o valor obtido no período pós-treinamento $(35 \pm 6 \mathrm{~cm})$ foi semelhante ao obtido pelos idosos participantes da oficina de equilíbrio no período pré-oficina. Assim, os valores obtidos e a não modificação significativa da medida no teste do alcance funcional pode ser explicados pelo fato de que todos os idosos avaliados já eram praticantes de atividade física orientada. Sabe-se que tais atividades, por recomendação médica para evitar o sedentarismo ou para melhorar a qualidade de vida, podem prevenir doenças e prolongar a vida ${ }^{25}$.

Um estudo realizado anteriormente chegou a conclusōes semelhantes ao expor que idosos ativos tinham menor propensão a queda do que indivíduos sedentários, pois apresentavam melhor equilíbrio, marcha e independência nas atividades de diárias ${ }^{26}$. A prática de atividade física orientada é essencial para a manutenção do equilíbrio, diminuindo a probabilidade de quedas $s^{8,27,28,29}$.

Assim, quanto ao teste do alcance funcional, verificouse que apesar de haver uma modificação na média dos valores obtidos, não foi significativamente superior no período pós-oficina de equilíbrio, provavelmente em funçáo da atividade física praticada pelos indivíduos avaliados.

Com relação ao medo de cair, é um problema comum. Está presente na maior parte dos idosos, mesmo naqueles que nunca caíram ${ }^{30}$. O medo pode gerar baixa confiança, fazendo com que se evitem algumas atividades da vida diária ${ }^{31}$, devendo ser pesquisado para que se definam estratégias para sua diminuição e para que o idoso não tenha problemas em desempenhar suas atividades rotineiras.

A análise dos resultados do instrumento FES-I demonstrou que, no período pré e pós-oficina, os valores obtidos foram inferiores aos obtidos por outros autores $^{32}$. Tais valores podem demonstrar que, provavelmente pela prática de atividade física, os idosos avaliados não apresentavam preocupação significativa com quedas, uma vez que a ausência de sedentarismo pode promover uma sensação de segurança, mesmo em ambientes mais propícios a quedas. Um estudo anterior demonstrou que o medo de cair está fortemente associado à redução de mobilidade e atividade física ${ }^{33}$, o que não é experimentado pelos participantes da pesquisa, uma vez que nenhum deles tinha qualquer restrição física.

Outro fato a ser destacado é o discreto aumento na pontuação do FES-I quando considerada a primeira e a segunda etapa da pesquisa. Este fato não era esperado pelos pesquisadores, e acredita-se que possa ser atribuído a um maior esclarecimento sobre os ambientes que podem contribuir para desencadear as quedas, tais como pisos escorregadios, subir e descer ladeiras, entre outros. Provavelmente alguns idosos não percebiam tais ambientes como sendo favorecedores de quedas e, após a oficina, ficaram mais atentos a atividades que poderiam originá-las, aumentando a preocupação. Este dado é reforçado por um estudo anterior, que demonstrou que os indivíduos praticantes de atividade física apresentavam valores mais altos no FES-I após um acompanhamento de prática regular de atividade física no período de 12 meses quando comparados com aqueles que não praticavam atividade física ${ }^{34}$. Assim, mesmo com poucos resultados, acredita-se que a realização da oficina foi importante, pois foi uma estratégia de promoção de saúde visando prevenir episódios de queda e, como consequência, auxiliando na manutenção da capacidade funcional, independência e qualidade de vida de idosos ${ }^{35}$.

Em função do número de idosos da amostra, optouse por não considerar, na análise de dados, o tipo de atividade física praticada. Sabe-se que esta é uma limitação do estudo, pois talvez o tipo de atividade física praticada influencie nas respostas aos testes aplicados. Acredita-se que esta lacuna possa ser preenchida em estudos futuros, com um maior número de participantes, onde a variável possa ser analisada. Pesquisas futuras deverão incluir também a análise dos resultados obtidos com idosos participantes da oficina de equilíbrio em comparação com resultados de idosos em um grupo controle. A adoção de grupo controle não foi incluída nesta pesquisa porque os dados foram obtidos em projeto de extensão que, posteriormente, originou um projeto de pesquisa.

Mesmo não havendo uma modificação significativa entre as avaliaçóes, acredita-se que os idosos foram beneficiados, uma vez que as atividades desenvolvidas provavelmente permitiram uma maior consciência corporal e levaram a alertas sobre a prevenção de quedas.

\section{Conclusão}

Verificou-se que a probabilidade de quedas e a preocupação em cair não foram modificadas significativamente com a realização de oficina de equilíbrio, 
provavelmente porque os idosos eram praticantes de atividade física orientada.

\section{Agradecimentos}

Os autores agradecem à Pró-Reitoria de Extensão da UFRGS, a FAPERGS e ao Programa de Extensão Universitária do Ministério da Educaçáo pelo incentivo à realização deste estudo na forma de concessão de bolsas de iniciação científica e extensão.

Os autores declaram que não possuem conflito de interesses econômicos, éticos e operacionais que comprometam a fidedignidade dos dados e sua isenção científica, tanto na análise como apresentação dos mesmos.

\section{Referências}

1. Rodrigues RAP, Kusumota L, Marques S, Fabricio SCC, Cruz IR, Lange C. Política nacional de atenção ao idoso e a contribuição da enfermagem. Texto Contexto Enferm. 2007;16(3):536-45.

2. Cruz DT, Ribeiro LC, Vieira MT, Teixeira MTB, Bastos RR, Leite ICB. Prevalência de quedas e fatores associados em idosos. Rev Saúde Públ. 2012;46(1):138-46.

3. Instituto Brasileiro de Geografia e Estatística. Censo demográfico 2010 e resultados preliminares. 2011.

4. Maciel ACC, Guerra RO. Prevalência e fatores associados ao déficit de equilíbrio em idosos. R Bras Ci e Mov. 2005;13(1):37-44.

5. Gerdhem P, Ringsberg KA, Åkesson K. The relation between previous fractures and physical performance in elderly women. Arch Phys Med Rehabil. 2006;87(7):914-17.

6. Gazzola JM, Perracini MR, Ganança MM, Ganança FF. Fatores associados ao equilíbrio funcional em idosos com disfunção vestibular crônica. Rev Bras Otorrinolaringol. 2006;72(5):683-90.

7. Ruwer SL, Rossi AG, Simon LF. Equilíbrio no idoso. Rev Bras Otorrinolaringol. 2005;71(3):298-303.

8. Ribeiro AP, Souza ER, Atie S, Souza AC, Schilithz AO. A influência das quedas na qualidade de vida de idosos. Ciên Saúde Colet. 2008;13(4):1265-73.

9. Celich KLS, Souza SMS, Zenevicz L, Orso ZA. Fatores que predispóem às quedas em idosos. RBCEH. 2010;7(3):418-25.

10. Xavier JJS. Equilíbrio em idosos e prática de tai chi chuan [dissertação]. Ribeirão Preto (SP): Universidade de São Paulo; 2008.

11. Fletcher PC, Hirdes JP. Restriction in activity associated with fear of falling among community-based seniors using home care services. Age Ageing. 2004;33(3):273-79.

12. Ishizuka MA. Avaliaçáo e comparação dos fatores intrínsecos dos riscos de quedas em idosos com diferentes estados funcionais [dissertaçáo]. Campinas (SP): Universidade Estadual de Campinas; 2003.

13. Ribeiro F, Teixeira F, Brochado G, Oliveira J. Impact of low costs strength training of dorsi- and plantar flexors on balance and functional mobility in institutionalized elderly people. Geriatr Gerontol Int. 2009;9(1):75-80.

14. Masud T, Morris RO. Epidemiology of falls. Age Ageing. 2001;30(4):3-7.

15. Gazzola JM, Muchale SM, Perracini MR, Cordeiro RC, Ramos LR. Caracterizaçáo funcional do equilíbrio de idosos em serviço de reabilitação gerontológica. Rev Fisioter Univ São Paulo. 2004;11(1):1-14.

16. Cruz E, Oliveira EM, Melo SIL. Análise biomecânica do equilíbrio do idoso. Acta Ortop Bras. 2010;18(2):96-9.

17. Rubira APFA, Silva MG, Carvalho TG, Sene M, Harakawa LSK, Rubira LA, Consolim-Colombo FM, Rubira MC. Efeito de exercícios psicomotores no equilíbrio de idosos. Cons Saude. 2013;13(1):54-61.

18. Mann L, Teixeira, CS, Pranke GI, Rossi AG, Lopes LFD, Mota CB. Equilíbrio estático de idosas praticantes de hidroginástica. Anais do XII Congresso Brasileiro de Biomecânica; 2007; Estância de São Pedro, Brasil. Rio Claro, Unesp, 2007.

19. Oliveira R, Magnani KO, Soares MML, Freitas ACM, Xavier TTN. Avaliação da auto-percepção do risco de quedas em idosos ativos. Ext Soc. [periódico na Internet]. 2011 [acesso em 1 maio 2014]; 3(3): . Disponível em http://ufrn. emnuvens.com.br/extensaoesociedade/article/view/1230

20. Camargos FFO, Dias RC, Dias JMD, Freire MTF. Crosscultural adaptation and evaluation of the psychometric properties of the Falls Efficacy Scale - International among elderly brazilians (FES-I-BRAZIL). Rev Bras Fisioter. 2010;14(3):237-43.

21. Duncan PW, Weiner DK, Chandler J, Studenski S. Functional reach: a new clinical measure of balance. J Gerontol. 1990;45(6):192-97.

22. Sacco ICN, Bacarin TA, Watari R, Suda EY, Canettieri MG, Souza LC, Oliveira MF, Santos S. Envelhecimento, atividade física, massa corporal e arco plantar longitudinal influenciam no equilíbrio funcional de idosos? Rev Bras Educ Fís Esp. 2008;22(3):183-91.

23. Oliveira MF. Análise do equilíbrio em idosos ativos: aplicação do teste do alcance functional. Rev Bras Educ Fís Esp. 2006;20(sup 5):635-62.

24. Neto AFL, Guimarães RF. Atividade física e incidência de quedas em idosos. Rev Saúde Desenvolvimento. 2012;1(2):28-43.

25. Ramos BMB. Influências de um programa de atividade física no controle do equilíbrio de idosos [monografia]. São Paulo (SP): Universidade de São Paulo; 2003.

26. Figliolino JAM, Morais TB, Berbel AM, Corso SD. Análise da influência do exercício físico em idosos com relação a equilíbrio, marcha e atividade de vida diária. Rev Bras Geriatr Gerontol. 2009;12(2):227-38. 
27. Guimarães LHCT, Galdino DCA, Martins FLM, Vitorino DFM, Pereira KL, Carvalho EM. Comparação da propensão de quedas entre idosos que praticam atividade física e idosos sedentários. Rev Neurociênc. 2004;12(2):11-5.

28. Rice J, Keogh JWL. Power training: can it improve functional performance in older adults? A systematic review. Int J Exerc Sci. 2009;2(2):131-51.

29. Rocha SPM. Efeitos do aumento da atividade física na funcionalidade e qualidade das pessoas idosas do Centro Social de Ermesinde [dissertação]. Lisboa: Universidade Técnica de Lisboa; 2012.

30. Dias RC, Freire MTF, Santos EGS, Vieira RA, Dias JMD, Perracini MR. Características associadas à restrição de atividades por medo de cair em idosos comunitários. Rev Bras Fisioter. 2011;15(5):406-13.

31. Rand D, Miller WC, Yui J, Eng JJ. Interventions for addressing low balance confidence in older adults: a systematic review and meta-analysis. Age Ageing. 2011;40(3):297-306.
32. Rodrigues IG, Costa GA, Pinto RMC. Qualidade de vida e senso de auto-eficácia para quedas em idosos participantes do projeto AFRID/UFU. EFDeportes. [periódico na Internet]. 2009. [acesso em 2 maio 2014]; 13(129):1. Disponível em http://www.efdeportes.com/efd129/qualidade-de-vida-e-senso-de-auto-eficacia-para-quedas-em -idosos.htm

33. Suzuki M, Ohyama N, Yamada K, Kanamori M. The relationship between fear of falling, activities of daily living and quality of life among elderly individuals. Nurs Health Sci. 2002;4(4):155-61.

34. Carvalho J, Pinto J, Mota J. Actividade física, equilíbrio e medo de cair. Um estudo em idosos institucionalizados. Rev Port Cienc Desp. 2007;7(2):225-31.

35. Menezes R, Bachion MM. Estudo da presença de riscos intrínsecos para a queda, em idosos institucionalizados. Ciênc Saúde Colet. 2008;13(4):1209-18. 